\section{A.004 SHARING TOOLS TO ENSURE A SAFE AND HEALTHY} JOURNEY TO SCHOOL

'Margaret Peden*, 'Prasanthi Puvanachandra, ${ }^{2}$ Agnieszka Krasnolucka. ${ }^{1}$ The George Institute UK, Oxford, UK; ${ }^{2}$ The FIA Foundation, London, UK

10.1136/injuryprev-2021-safety. 151

Context Each day millions of children worldwide face the risk of road traffic injury and dangerously toxic air on their journey to access education. But we have the solutions, the tools to combat road traffic injury and ensure our children can breathe clean air. The solutions are readily available and affordable, bringing results that are measurable.

Process The Child Health Initiative - a group of 20 organizations - is hosted by the FIA Foundation. Its mission is to ensure a safe and healthy journey to and from school for every child by 2030. The Initiative combines research, advocacy and evidence-based interventions in order to promote this child rights agenda.

Analysis The George Institute, together with the FIA Foundation, conducted an assessment of available safe routes to school projects being conducted around the world and the tools being used.

Outcomes An online toolkit was constructed, see https:// www.childhealthinitiative.org/toolkit. It covers: (a) Data collection (b) Implementation and Design (c) Coalition building and (d) Achieving impact. Tools, resources, case studies and evidence were collated in a user-friendly, dynamic platform in order to provide guidance to those wishing to undertake similar research or programmes in countries around the world.

Learning Outcomes NGOs, international agencies, public authorities and their partners around the world will be able to find step by step guidance within the toolkit to help implement the solutions. This resource supports organisations aiming to create a safe environment for children, to protect them from air pollution and prevent child road traffic injury.

\section{B - Drowning - Data, March 25, 2021}

\section{B.001 USING DEATH CERTIFICATE LITERAL TEXT TO IDENTIFY THE LOCATION OF DROWNING DEATHS}

Holly Hedegaard*, Merianne Rose T Spencer, Margaret Warner. National Center For Health Statistics, Bethesda, USA

\subsection{6/injuryprev-2021-safety. 152}

Background ICD-10 codes provide limited information on the location of drowning deaths, particularly those occurring in natural water. Application of natural language processing (NLP) to literal text on death certificates can provide greater detail.

Methods Records that include literal text were selected from 2011-2017 U.S. National Vital Statistics System mortality data using ICD-10 multiple cause codes W65-W74, V90, V92, X71, X92, Y21 and T75.1. SAS Contextual Analysis software was applied to literal text data from six fields on the U.S. standard death certificate to create a list of possible terms describing drowning locations. Terms were categorized as: Domestic (e.g., swimming pool, bathtub, spa), NonDomestic Freshwater (e.g., lake, pond, river, reservoir, well), Saltwater (e.g., ocean), Other, and Unspecified. For Non-
Domestic Freshwater, subcategories included Natural and Man-made.

Results Of 26,638 drowning deaths, 32\% were categorized to Domestic, $52 \%$ to Non-Domestic Freshwater, 10\% to Saltwater, and $6 \%$ to Other or Unspecified. For Non-Domestic Freshwater, $90 \%$ occurred in natural settings and 10\% involved a man-made source (e.g. well, reservoir, cistern, tank). Among the Natural Non-Domestic Freshwater drownings, about half $(52 \%)$ occurred in still water (e.g., lake, pond, wetlands, marsh) and half (47\%) occurred in moving water (e.g., river, creek, rapids, flooding).

Conclusion Application of NLP methodology can provide more detailed information on location of drowning deaths. This information can be used to target prevention efforts to high risk populations by location.

Learning Outcomes To learn about the application of NLP processing to death certificate literal text to identify and categorize locations of drowning deaths.

\section{B.002 EXPLORING CHILD DROWNING PREVENTION ACROSS AUSTRALIA, CANADA AND NEW ZEALAND}

\footnotetext{
${ }^{1,2,3}$ Amy Peden*, ${ }^{2,3}$ Richard Franklin, ${ }^{4}$ Tessa Clemens. ${ }^{1}$ School of Public Health and Community Medicine, University of New South Wales, Kensington, Australia; ${ }^{2}$ Royal Life Saving Society - Australia, Broadway, Australia; ${ }^{3}$ College of Public Health, Medical and Veterinary Sciences, James Cook University, Townsville, Australia; ${ }^{4}$ Drowning Prevention Research Centre Canada, Toronto, Canada
}

\subsection{6/injuryprev-2021-safety. 153}

Background Young people are the leading contributor to the global drowning burden with children under five at highest risk. This study explored temporal trends in fatal child drowning across three high income countries: Australia, Canada and New Zealand.

Methods A total population analysis of all unintentional drowning deaths among 0-19 year olds from 2005-2014 was undertaken using data from national drowning prevention organisations from each country.

Results 1,454 children and adolescents $(17.8 \%$ of all drowning deaths) died from drowning across the three countries; rates ranged from 0.92 (Canada) to 1.35 (New Zealand) per 100,000. When examining linear trends over time of crude drowning rates, both Australia $(y=-0.041)$ and Canada $(y=-$ $0.048)$ reduced with New Zealand $(y=0.005)$ showing a slight increase, although highly variable year-to-year. Reductions in all three countries have been seen at rivers and swimming pools. For New Zealand drowning deaths while boating and in lakes/ponds have increased.

Conclusions Investment in prevention in Australia and Canada is showing results. Lessons from each country may help enhance drowning prevention across the three countries and other high-income contexts. Further research is needed to definitively confirm effectiveness of preventative strategies such as mandating pool fencing through legislation and enforcement, bystander CPR and increased public awareness.

Learning Outcomes Routinely collated data with consistently reported variables allows comparison between and across countries. This study highlights where achievements have been made in the reduction of unintentional fatal child drowning to strengthen investment in existing prevention strategies or to encourage investment or policy change where needed. 